\title{
RETOS EDUCATIVOS PARA LA CONVIVENCIA EN IGUALDAD
}

\author{
Ana Agirre Sáez de Eguilaz \\ Diputación Foral de Gipuzkoa
}

RESUMEN: El sistema sexo-género, según el cual se asocian unos roles y una posición social determinada a mujeres y hombres en función del sexo de pertenencia, determina la construcción de nuestra identidad personal y social y condiciona enormemente nuestros modelos de convivencia $y$, en general, toda la organización social.

Sin embargo, y en la medida en que este modelo de socialización no es sino un constructo cultural, es susceptible de ser modificado. En este sentido, la educación constituye un marco fundamental para promover la igualdad de mujeres y hombres desde la intervención planificada e intencionada. El objetivo sería, lograr el desarrollo integral de niños y niñas, a fin de que construyan sus relaciones adultas futuras desde un modelo más justo e igualitario, es decir, desarrollando todas las facetas de su vida (personal, social, familiar y laboral) desde la autonomía y la corresponsabilidad.

Palabras clave: Socialización de género, desarrollo integral, coeducación, igualdad.

\section{EDUCATIONAL CHALLENGES TO LIVE TOGETHER IN EQUALITY}

ABSTRACT: Gender system assigns roles and a particular social position to women and men, depending on their gender. This greatly influences the model of coexistence between women and men and, in general, social organization as a whole. In fact, gender socialization creates a bias in the development of people and also ranks the models assigned to men and women, giving greater value and appreciation for everything associated with men and masculinity.

However, this model of socialization is not but a cultural construct, so that it is susceptible to be modified. In this sense, education is a basic framework 
to promote equality between women and men. The aim would be to achieve the integral development of boys and girls in order to build their future adult relationship from a more and egalitarian model. This way they would be able to approach all facets of life (personal, social, family and work) from full autonomy and sharing responsibilities.

Keywords: Gender socialization, integral development, coeducation, equality.

\section{Contextualización}

Los modelos de convivencia que rigen nuestras vidas actuales son fruto de los procesos de construcción de la identidad personal colectiva que mujeres y hombres hemos vivido. Y para su comprensión se hace imprescindible el análisis de la categoría género y su incidencia tanto en la convivencia cotidiana de mujeres y hombres, como en la organización social en general.

De este modo las personas, a lo largo del proceso de socialización, van desarrollando distintas capacidades e interiorizando diferentes valores, actitudes y comportamientos, es decir, en función de su sexo, hombres y mujeres son adscritos a un género determinado. El género se convierte así en una categoría social que define las expectativas diferentes que la sociedad tiene para unas y otros. Es lo que se conoce como socialización genérica.

Tradicionalmente se ha socializado a las mujeres para que asuman responsabilidades y tareas relacionadas con la familia y el hogar, mientras que a los hombres se les ha educado para proveer a esa familia y ese hogar de los recursos económicos necesarios para su mantenimiento. Es decir, a ellas se les "diseñaba" para un futuro en el mundo doméstico y reproductivo y a ellos para el mundo público y productivo.

Evidentemente, este modelo tradicional de socialización ha evolucionado y en la actualidad no se realiza de un modo tan segregado y diferenciado. De hecho, en las últimas décadas se ha producido una incorporación masiva de las mujeres al ámbito laboral y esto ha puesto en cuestión los modelos tradicionales de independencia-dependencia económica y con ello, los modelos de convivencia en el marco de la pareja. Además, desde hace ya cuatro décadas, en nuestro contexto chicas y chicos son educados juntos, compartiendo espacios y contenidos escolares. Y unos y otras son preparados para desenvolverse en el ámbito público.

Sin embargo, la socialización de género sigue produciéndose a través de mecanismos más sutiles. Aunque niños y niñas comparten espacios de socialización, reciben constantemente mensajes y modelos diferentes de cómo debe ser un hombre y cómo debe ser una mujer. Tanto la familia, como la escuela, los medios y el entorno permiten, alientan, refuerzan o penalizan determinadas actitudes y comportamientos en los chicos y otros diferentes en las chicas. 
De este modo sigue produciéndose una socialización diferente de las personas en función de su sexo. Y esto genera una parcialización de las personas en su desarrollo. Todavía a las niñas se les potencia más aquellas capacidades, valores y actitudes tradicionalmente asociadas a la "feminidad" (sensibilidad, ternura, belleza abnegación, entrega...), mientras que a los niños se les potencia más aquellas capacidades, valores y actitudes asociadas a la "masculinidad" (iniciativa, autoafirmación, competitividad, seguridad, agresividad, independencia...).

Este modelo diferente de socialización para niñas y niños no genera una mera segregación en el desarrollo, con la consecuente parcialización para unas y otros. Además de una separación produce una jerarquización, de modo que socialmente son más valoradas las capacidades, valores y actitudes asociadas al mundo masculino, que las asociadas al mundo femenino.

E incluso persisten mecanismos que contribuyen al mantenimiento de las desigualdades en aquellos ámbitos aparentemente más igualitarios como sucede con la educación. Así, por ejemplo, en la escuela se prepara tanto a las chicas como a los chicos para desenvolverse en el ámbito público, es decir, para ejercer una profesión, y para la participación social y política. Sin embargo, la esfera tradicionalmente asociada a lo femenino (trabajo doméstico y de cuidado) queda al margen de esta formación, reforzando con ello su invisibilización y su devaluación, a pesar de ser un trabajo imprescindible para el sostenimiento de la vida humana.

En consecuencia, está mejor asumida socialmente la "masculinización" de las mujeres que la "feminización" de los hombres, a los que rápidamente se les etiqueta como "raros" o "débiles". Es decir, se acepta mejor la incorporación al modelo valorado socialmente (el masculino) que la adscripción al modelo devaluado (el femenino).

Por ello, las chicas, en su afán de igualdad, cada vez van adoptando más actitudes y comportamientos considerados tradicionalmente como masculinos (lenguaje, deporte, aficiones, actitudes de riesgo, comportamientos violentos, escasa vinculación al trabajo doméstico y de cuidado, etc.).

Este proceso va produciéndose sutilmente, de modo que casi no lo percibimos y cuando lo hacemos nos parece "natural". Y de este modo, la jerarquización entre el mundo masculino y el femenino comienza a construirse desde las primeras relaciones infantiles. Y es que en la familia y en la escuela, los niños y las niñas no sólo aprenden a convivir, también aprenden los roles de dominantes y dominadas respectivamente. De hecho, muchos de los problemas de convivencia que se manifiestan en la escuela tienen su origen en los modelos de relación y de convivencia construidos en función de los roles de género.

Sin embargo, como este modelo es fruto de una construcción cultural, puede ser analizado, revisado y modificado en aras a construir nuevos modelos de convivencia basados en el respeto, la igualdad y la diversidad. Para ello es 
necesario conocer los mecanismos de actuación de los diferentes agentes de socialización y actuar sobre ellos.

Una breve aproximación a los ámbitos fundamentales de socialización, nos permite tomar conciencia del papel que pueden llegar a jugar en la legitimación y reproducción de las desigualdades de género:

\subsection{Familia}

La familia es el primer contexto de socialización de la criatura. En función de los modelos de relación ofrecidos especialmente por las personas adultas responsables de su cuidado, el niño o la niña irá interiorizando y aprendiendo valores y comportamientos que trasladará a sus futuras relaciones.

En el entorno familiar las criaturas reciben constantemente mensajes, no sólo a través de lo que oyen, sino también y fundamentalmente a través de lo que ven. Es decir, básicamente se educa a través de lo que se hace, de lo que se es, no de lo que se dice. Y todavía los modelos que las personas adultas de referencia ofrecen a las criaturas están fuertemente condicionados por los roles de género.

De este modo, desde pequeñitos y pequeñitas van percibiendo que lo que hacen, dicen y sienten las mujeres tiene un reconocimiento menor que lo que hacen, dicen o sienten los hombres. De este modo, los niños van aprendiendo a hacerse valer desde la imposición, la fuerza, la autoafirmación y las niñas van aprendiendo a asumir su propia inferioridad y a mantenerse en un segundo plano o bien comienzan a imitar el mundo masculino para lograr con ello cierto reconocimiento. Así, desde edades tempranas los niños aprenden que sus deseos, intereses y necesidades son más valorados por el entorno, mientras que las niñas aprenden a renunciar a los intereses, deseos y necesidades propias. $Y$ esto se manifiesta tempranamente en el tipo de juguetes que seleccionan, en el papel que asumen en los juegos, en las actitudes que manifiestan en los mismos, en la ocupación que realizan de los espacios, etc.

\subsection{Medios de comunicación}

Los medios de comunicación también ejercen una gran influencia en el modo de interpretar la realidad y en la construcción de los modelos de masculinidad y de feminidad. Especialmente la televisión, que forma parte de la vida cotidiana de nuestros niños y niñas, es un elemento de gran potencia en la transmisión y construcción de estereotipos.

De este modo, fundamentalmente son hombres los protagonistas de la realidad que presentan los medios de comunicación, tanto en la ficción como en la información. Y esto no hace sino reforzar la posición de superioridad del modelo masculino. 
Por otro lado, medios tan potentes como la televisión y el cine presentan constantemente múltiples formas de violencia, mayoritariamente ejercida por los hombres, en estrecha relación con los modelos de género. Así, en los medios, son múltiples las manifestaciones de violencia que perciben las niñas y los niños: violencia verbal, violencia callejera, violencia en el deporte, violencia bélica, violencia en la carretera, etc. Y además, pronto van tomando conciencia de que estas manifestaciones de violencia van acompañadas de un cierto prestigio social. La violencia es objeto de conversaciones y ocupa un espacio importante en los medios de comunicación. Además, a menudo va asociada a roles protagonistas y al liderazgo, por lo que hasta puede ejercer cierto efecto atractivo.

Por el contrario, las actividades relacionadas con el cuidado y el mantenimiento de la vida no tienen esos espacios de protagonismo. $Y$ de este modo, nuevamente el modelo masculino queda sobredimensionado, frente a un modelo femenino cuyas aportaciones quedan infravaloradas.

\subsection{Escuela}

La escuela como un ámbito más de la sociedad, refleja esta situación. No es la responsable de la misma, ni tiene todo el poder para modificarla. Sin embargo, su capacidad de intervención es muy importante, en la medida en que constituye el espacio formal de educación por excelencia y además, acoge a todas las criaturas, sea cual sea su condición y procedencia.

En la actualidad, cada vez es mayor la preocupación de la comunidad escolar por la cuestión de la convivencia escolar. Y esto está muy relacionado con las manifestaciones de violencia y agresividad en los centros escolares.

Sin embargo, todavía las intervenciones están muy centradas en el establecimiento de normas y sanciones y resulta difícil intervenir incorporando criterios para determinar el límite entre las actuaciones y comportamientos aceptables y los agresivos.

Consecuentemente existen conductas y actitudes "normalizadas' que no se consideran agresivas ni se asocian con un modelo de socialización basado en el género. Por ello, continúa en gran medida aceptándose como normal que los juegos practicados por los alumnos incorporen acciones físicas como insultos, empujones, patadas, peleas, etc. No obstante, la escuela tiende a reprimir más estos mismos comportamientos en las chicas.

Igualmente, en la escuela cada niña y cada niño recibe determinadas visiones de la realidad y modelos de relación, a menudo fuertemente condicionados por los mandatos de género. También aquí aprenden que todo aquello que no se reconoce ni valora socialmente forma, mayoritariamente, parte del modelo femenino y esto fomenta que a menudo los chicos se sientan superiores e infravaloren o incluso desprecien lo que dicen y hacen las chicas. Y todo 
esto va creando las condiciones necesarias para la utilización de la agresión, física, verbal o simbólica, en función del género, en la escuela y en otros ámbitos de la vida.

\section{Análisis de la situación: niñas y niños en la escuela}

Un acercamiento a cualquier escuela y la simple observación de las interacciones que se producen entre chicos y chicas, tanto en los espacios formales (aula, actividades dirigidas, etc.) como en los informales (pasillos, entradas y salidas, recreo, comedor, etc.), nos permite realizar una aproximación a la incidencia del género en los modelos de relación y convivencia vigentes.

Por otro lado, el análisis sencillo y cercano de sus concepciones sobre sí mismos y sí mismas y de sus expectativas vitales nos ayuda a identificar los modelos de género que guían sus deseos y anhelos.

\subsection{La convivencia en la escuela}

Las escuelas de nuestro entorno viven diariamente este tipo de situaciones, a menudo consideradas como "normales" precisamente por su cotidianeidad.

Del análisis de estas situaciones se despenden algunas conclusiones muy interesantes, tal como se recoge en la guía elaborada en el marco del proyecto europeo "PAREKO: Hacia una escuela coeducadora"1. De hecho, la observación cualitativa de las interacciones en el aula, refleja que la mayoría de los problemas de convivencia en los centros están asociados al género.

Estos son algunos de los aspectos más significativos que se desprenden del análisis realizado en el marco de dicho proyecto:

Uso de la palabra y protagonismo de chicas y chicos

En términos generales, el uso de la palabra y el protagonismo, la voz y el espacio son, fundamentalmente, de los chicos. Estos hablan y participan bastante más veces que las chicas. Y además de hablar, los chicos gesticulan, se mueven, interrumpen, comentan entre sí e incluso gritan mucho más que las chicas.

Es decir, en general, los chicos ocupan el espacio verbal, físico y simbólico en la escuela.

\section{Conflictos de convivencia}

La mayor parte de los conflictos de convivencia existentes en los centros escolares de nuestro entorno son protagonizados por chicos. Evidentemente,

1. "Guía de buenas prácticas PAREKO", Ed. Emakunde/Instituto Vasco de la Mujer, VitoriaGasteiz, 2000. 
no todos los chicos generan conflictos, pero cuando éstos se producen, sus protagonistas son fundamentalmente chicos.

Sin embargo, existe escasa conciencia de ello entre el profesorado y, por lo tanto, no se actúa en consecuencia. Incluso a menudo tiende a responsabilizarse de los actos a todo el grupo, actuando indiscriminadamente sobre él.

Consecuentemente, estas conductas negativas para la convivencia se ven incluso reforzadas, ya que no van acompañadas de la correspondiente asunción de responsabilidades. Y con ello, los valores asociados a la masculinidad hegemónica que las sustentan se refuerzan, mientras que se ahonda en el papel sumiso de las chicas y de los chicos que no se adscriben a ese modelo de masculinidad. Es decir, una vez más, se potencian valores femeninos ligados a la sumisión y a la renuncia de los propios intereses y derechos para apoyar los intereses de "otros".

\section{Chicos que se pasan y chicas que pasan}

El papel protagonista de los chicos Ilega en algunos casos a la prepotencia y chulería de algunos de ellos, que manifiestan comportamientos condescendientes para con las chicas, a base de risitas, bromas de dudoso o mal gusto, interrupciones constantes a sus intervenciones, comentarios de infravalorización, etc.

Ante esta situación mayoritaria de preponderancia de los chicos y de lo masculino, la actitud de las chicas es, en general, de desánimo. Creen que no hay nada que hacer y es inútil esforzarse en hablar de ello con sus compañeros o con el profesorado. Consideran que nada cambiará y que intentarlo es sólo una pérdida de tiempo y energía.

No se sienten bien en esta situación, pero la aceptan sumisamente como inevitable e inmodificable. Por ello, renuncian incluso a solicitar la intervención de personas adultas.

Y con ello, se mantiene la jerarquización de género que prepara el terreno para las relaciones de dominación-sumisión.

\section{Generalización del modelo masculino}

Un fenómeno generalizado es la universalización o generalización del modelo masculino tradicional, de forma que sus características y sus valores se difunden y presentan como ideales tanto para los chicos como para las chicas.

Simultáneamente, a menudos se observa un cierto desprestigio y desvalorización de los valores y actitudes del modelo femenino tradicional, cuyas características se presentan como cargas y condicionantes indeseables tanto para chicas como para chicos.

De este modo, el androcentrismo constituye el eje estructurador de la organización y la vida escolar: las decisiones se toman en función de las ne- 
cesidades e intereses de los chicos y posteriormente, se "invita" a las chicas a ser partícipes de ellas.

Según expresan las propias chicas, está bien visto que "las chicas sean como chicos sin pasarse mucho". Parece que se les anima y se les aplaude si, por ejemplo, juegan al fútbol, si se integran en las aficiones y modos de relación de sus compañeros o si desarrollan sus estilos de camaradería. Pero, al contrario no se potencia, sino que se censura directamente, el hecho de que los chicos se integren en los juegos, costumbres, aficiones o modos de relación de las chicas.

Y con ello, una vez más, se imponen en el ámbito público los valores e intereses masculinos, no sólo para los chicos sino también para las chicas, ya que continúan siendo los de mayor prestigio social.

\section{Transgresiones}

En muchos centros se observan que hay chicos que no responden al modelo mayoritario de sus compañeros, es decir, no responden al modelo hegemónico de masculinidad.

Estos chicos, salvo algunos casos de excepcional carácter y personalidad, se encuentran marginados y sufren frecuentes humillaciones y desprecios por parte de sus compañeros.

No son aceptados porque sus actitudes y comportamientos son identificados como femeninos y esto es algo "intolerable" en un chico, ya que la masculinidad se construye desde la negación de la feminidad.

\subsection{Autoimagen}

También es importante analizar la imagen que de sí mismas y de sí mismos tienen las chicas y los chicos. Indagar en sus propias concepciones permite observar la fuerza con que los estereotipos de género siguen actuando en la construcción de la identidad personal de unas y otros.

De este modo, vemos que cuando se les pregunta a las niñas como se ven a sí mismas o como consideran que una chica debe ser, en sus respuestas utilizan adjetivos como los siguientes:

\section{"ordenadas, generosas, buenas, simpáticas, amables, responsables, tranquilas"}

Por su parte los chicos, se definen con otro tipo de calificativos:

$$
\begin{aligned}
& \text { "fuertes, hábiles, atrevidos, traviesos, } \\
& \text { inteligentes, vagos" }
\end{aligned}
$$


La diferente elección de adjetivos que realizan unas y otros para definir cómo debe ser una chica o un chico nos ayuda a entender los modelos de identidad desde donde se están construyendo niños y niñas y que indudablemente aluden, en gran medida, a los estereotipos de género interiorizados.

Además, nos invita a reflexionar sobre la necesidad de generalizar aspectos altamente valiosos de la feminidad como la responsabilidad y la generosidad, por ejemplo, así como de intervenir sobre algunos aspectos de la masculinidad que podrían estar incidiendo, por un lado en los peores resultados académicos ("vagos") y por otro en las relaciones de dominación ("fuertes") y la asunción de riesgos, ("atrevidos") innecesarios.

\subsection{Expectativas de futuro}

Por último, es necesario analizar las expectativas de futuro que están construyendo chicas y chicos, y que incidirán en sus futuras vidas adultas, ya que en estas proyecciones se comienza a dibujar las diferentes posiciones sociales que se atribuyen a sí mismos y a sí mismas.

En relación con la elección profesional, en general las chicas eligen profesiones consideradas tradicionalmente como femeninas y que se caracterizan bien por ser una especie de prolongación en el ámbito laboral del rol asignado a las mujeres en el marco del hogar y la familia como cuidadoras, servidoras y educadoras:

"maestra", "cuidadora", "enfermera", "camarera"

o bien por mantener la posición de subordinación de las mujeres

"secretaria", "enfermera"

o por su relación con el ámbito de la estética, la belleza y el cuerpo

"modelo", "peluquera"

Por su parte los chicos, se decantan por profesiones consideradas tradicionalmente masculinas,

"fontanero", "carpintero", "camionero", "bombero", "médico" 
destacando la relevancia del ámbito deportivo en la constitución de sus expectativas de vida adulta.

\section{"futbolista", "deportista"}

El fútbol y el deporte es para la mayoría de los chicos un referente de gran influencia. A menudo proyectan sus expectativas de éxito en sus ídolos deportivos, los cuales son asociados al éxito y al dinero.

En este sentido, cabe destacar que mientras a menudo las chicas esperan obtener éxito y reconocimiento social a través de su cuerpo, de su apariencia física, los chicos esperan obtenerlo a través del dinero y la carrera profesional.

Una vez más, el ámbito profesional, y el dinero obtenido a través de él, aparecen como referente masculino, mientras que en el imaginario de las chicas se mantiene el deseo de gustar y atraer a esos hombres de éxito. No es difícil intuir que tras el deseo de posesión material de los chicos y el deseo de gustar de las chicas se dibuja nuevamente la sombra de las relaciones de dominación y sumisión.

Mientras para los chicos una chica guapa y atractiva es un elemento "material" más que indica éxito social, para las chicas los deseos propios se desvanecen, ya que su deseo no es sino ser deseadas, es decir, responder a los deseos del otro, con lo que vuelven a posicionarse en un rol sumiso.

Finalmente, cabe destacar otro aspecto aún más interesante en relación con las expectativas de vida adulta.

Las chicas, por su parte, parecen proyectar su vida hacia tres ámbitos fundamentales: familia, profesión y tiempo propio. De este modo, les gustaría desarrollar estos tres aspectos de su vida de modo equilibrado. $Y$ en caso de no poder hacerlo, ponen en cuestión su futura maternidad.

Sin embargo, los chicos se proyectan fundamentalmente hacia lo profesional y lo personal, es decir, anhelan éxito profesional y desean mantener amistades y aficiones. Y para conseguirlo están dispuestos a dedicar esfuerzo y tiempo. Respecto al ámbito familiar se proyectan a sí mismos viviendo en pareja y siendo padres, pero no parece que estén dispuestos a dedicar su tiempo y esfuerzo al cuidado de las hijas e hijos o al cuidado del hogar. Los chicos reconocen el papel y el valor del trabajo reproductivo en sus futuras vidas adultas, pero no parecen mostrar excesivo interés en compartirlo con las mujeres.

Vemos, de este modo, que estos posicionamientos ante la vida doméstica y familiar condicionarán las futuras relaciones de pareja del alumnado actual, las cuales difícilmente podrán construirse de modo igualitario, si no realizamos las correspondientes intervenciones educativas.

Este es un dato muy relevante porque nos indica la necesidad de abordar el tema de la corresponsabilidad en el ámbito familiar y doméstico. Si los chicos no 
asumen su responsabilidad en el mismo, difícilmente las chicas podrán alcanzar el desarrollo equilibrado que anhelan entre familia, profesión y tiempo propio.

Y lo que aún es más grave, las parejas que entre ellas y ellos surjan volverán a construirse sobre esquemas de dominación y subordinación, ya que se basarán en modelos jerarquizados de feminidad y masculinidad.

Vemos así que cuando indagamos sobre las actitudes y comportamientos del alumnado, su autoimagen y sus expectativas de futuro, se dibujan caminos diferentes para chicas y chicos en función de los modelos hegemónicos de masculinidad y feminidad. Caminos que a su vez aparecen definidos por esquemas relacionales de dominación y sumisión que son los que posibilitan la violencia de género. Violencia que afecta tanto a las relaciones actuales entre chicas y chicos y que constituye la base sobre la que construirán sus futuras relaciones de pareja.

Aún cuando la gente joven de nuestro entorno mantiene una posición generalizada favorable a la igualdad de mujeres y hombres, no son conscientes de las desigualdades existentes. La sutileza de las mismas les Ileva a vivir en un falso "espejismo de igualdad", es decir, consideran que la igualdad ya es algo conseguido, algo por lo que no hay que luchar.

Sin embargo, esta creencia no tiene un reflejo en la práctica, ya que la vida escolar y social, y en general las relaciones entre chicos y chicas, todavía está fuertemente ligada a los modelos de género y con ello, se sigue reproduciendo desigualdades entre unas y otros.

\section{Implicaciones educativas}

La primera implicación educativa que se deriva de este análisis es que sólo desde la intervención intencionada se producirán cambios hacia la igualdad. Los estereotipos y las relaciones jerárquicas de género son enormemente potentes, por lo que si no se interviene sobre ellos tienden a mantenerse y reproducirse.

Cualquier programa de educación para la convivencia debería tener en cuenta todo esto. Para ello, habría que fomentar el análisis y el dialogo entre el propio profesorado y con el alumnado y con las familias sobre las manifestaciones de los estereotipos de género tanto en el ámbito familiar como escolar y social. Igualmente habría que escuchar, reconocer y valorar la palabra, las necesidades, las experiencias y las formas de percibir el mundo tanto de chicas como de chicos.

No se trata tanto de intentar resolver los conflictos, inherentes a toda relación humana, a través de un exceso de normas e interpretaciones ajenas a la propia realidad de quienes se ven afectados y afectadas por ellos, sino de fomentar actitudes de rechazo ante cualquier situación de desprecio, infravaloración o agresión.

Esto favorecerá que las niñas, con frecuencia víctimas de las agresiones, dejen de asumirlas de forma resignada. Al mismo tiempo, los niños interiorizarán que para ser valorados y reconocidos socialmente no necesitan hacer uso de la prepo- 
tencia y la fuerza. Y todo ello contribuirá a "desmontar" toda la mística de la masculinidad que todavía hoy juega un papel importante en nuestra tradición cultural.

El logro de un clima positivo en los centros educativos, donde chicas y chicos participen en condiciones de igualdad y convivan en un ambiente de respeto y dialogo, pasa por el análisis de los diferentes procesos de socialización de niñas y niños en función de su sexo.

Es necesario analizar y reflexionar sobre los mecanismos de socialización genérica para desentrañar los diferentes valores y actitudes que chicas y chicos han desarrollado en función del rol de género al que se han visto adscritas y adscritos. Sólo así podremos tomar conciencia de la incidencia de los valores de género interiorizados en las diferentes actitudes y comportamientos de chicas y chicos. Y esta toma de conciencia nos permitirá diseñar estrategias adecuadas de intervención.

Sólo desde un diagnóstico cualitativo de los modelos de relación entre chicas y chicos podremos buscar nuevas vías que contribuyan a una convivencia justa y democrática, que contribuya al reconocimiento y al desarrollo pleno tanto de niñas como de niños.

En este contexto, se hace necesario trabajar intencionalmente la coeducación, ya que sólo desde la construcción de unas relaciones igualitarias entre chicas y chicos se podrá mejorar la convivencia en los centros y, a su vez, prevenir la violencia contra las mujeres en las futuras relaciones de pareja.

Pero trabajar la coeducación, no quiere decir educar en igualdad, sino educar para la igualdad. Esto, supone trabajar aspectos diferentes con niños y con niñas ya que han vivido procesos de socialización diferentes, que hacen que sus posicionamientos en las relaciones y en la vida en general sean diferentes.

Y para ello, es necesario intervenir en algunos ámbitos específicos en relación con los chicos y en otros en relación con las chicas.

\section{1. Ámbitos de intervención con chicos}

Con los chicos habría que trabajar fundamentalmente tres cuestiones:

\subsubsection{Masculinidad}

Se trataría de romper los nexos entre violencia y masculinidad a fin de fomentar nuevos modelos de masculinidad que no vayan asociados a la agresividad, la trasgresión e irresponsabilidad frente a la norma y la intolerancia hacia quien es diferente y se percibe como más débil.

Con ello, contribuiríamos a la construcción de nuevos modelos de convivencia, a la resolución pacífica de los conflictos y al respeto a las diferencias de género y a otras diferencias (étnicas, culturales, sexuales, de edad, etc.). 
Además, el curriculum debería incorporar aspectos relacionados con el conocimiento del funcionamiento de las relaciones interpersonales. Esto contribuiría a conocer los aspectos de género relacionados con las mismas y que están condicionando las relaciones chicos-chicas y especialmente la relación de pareja, ya que todavía el papel de los hombres se asocia al dominio, a la satisfacción de sus necesidades y deseos y el de las mujeres a la dependencia, la sumisión y la satisfacción de las necesidades y deseos de los demás.

\subsection{2. Ética del cuidado}

El cuidado forma parte constante de nuestra vida cotidiana. Por ello, es necesario que desde el contexto escolar se fomente la ética del cuidado, especialmente en los chicos, a fin de que vayan desarrollando habilidades, actitudes y comportamientos que contribuyan a establecer otros modelos de relación menos dominantes y destructivos.

De este modo, se hace necesario incidir sobre aspectos relacionados con el autocuidado, el cuidado de personas y el cuidado del entorno. Con ello contribuiremos a deconstruir el estereotipo de la masculinidad, fomentando mayores niveles de humanización de las relaciones y mayores niveles de corresponsabilidad entre mujeres y hombres.

\subsubsection{Corresponsabilidad}

Se trataría de integrar en el curriculum escolar aspectos relacionados con la vida doméstica y familiar, a fin de que las chicas, y muy especialmente los chicos, desarrollen habilidades, actitudes y comportamientos de colaboración que contribuyan a una auténtica corresponsabilidad en el hogar, tanto en el momento actual como en su futura vida adulta.

Sólo así, podrá crearse el contexto adecuado para que mujeres y hombres se integren en igualdad de condiciones en la vida laboral y social y todos y todas sean partícipes y responsables de las responsabilidades domésticas y de cuidado.

\section{2. Ámbitos de intervención con chicas}

Por su parte, con las chicas habría que trabajar en torno a un eje fundamental: el empoderamiento ${ }^{2}$. Se trataría de iniciar procesos de "fortalecimiento" de las niñas para ayudarles a conseguir:

2 El término empoderamiento es una traducción literal del concepto inglés "empowerment" y significa "ganar poder", fortalecerse. Alude a un proceso de toma de conciencia individual y colectiva de las mujeres, que les permite aumentar su participación en los procesos de toma de decisiones y de acceso al ejercicio de poder y a la capacidad de influir. 


\subsubsection{Autonomía física y emocional}

Desarrollar la autonomía física y emocional supone trabajar su propia autoestima y la asertividad a fin de que aprendan a escuchar y atender sus propios deseos y dejen de construirse para satisfacer los deseos ajenos.

\subsubsection{Autonomía económica}

La autonomía económica es fundamental para construir relaciones afectivas desde la igualdad y no desde la dependencia.

Para desarrollarla es necesario trabajar el tema de la centralidad del empleo. El empleo debe ser algo incuestionable en las chicas y no una opción, como todavía continua siendo en el imaginario colectivo. El empleo es lo que nos posibilita la autonomía económica, que es imprescindible para la autonomía personal. Por ello, nunca debe ser algo cuestionable o que se relega a un segundo plano en función de las necesidades del ámbito familiar y doméstico. En la medida en que lo sea, las chicas se situarán en una posición de dependencia y de subordinación y con ello, de sumisión.

\subsubsection{Autonomía personal}

Se trata de incidir con las chicas en la necesidad de tener un proyecto de vida propio. Su único proyecto no puede ser servir al proyecto de otras personas, porque supone la renuncia absoluta a los propios intereses y deseos y conlleva la propia anulación.

Por eso se hace necesario que cada persona tenga su propio proyecto de vida y la relación de pareja, en el caso de construirla, debería apoyar ese proyecto y realizar los ajustes necesarios para que los proyectos de las dos personas que la conforman puedan desarrollarse.

En definitiva, se trata de que la escuela fomente el desarrollo integral de chicas y de chicos. Y para ello es necesario que el ámbito educativo reconozca y valore por igual los intereses, experiencias y saberes tanto de hombres como de mujeres.

Sólo desde un desarrollo integral de nuestras alumnas y alumnos que les prepare para afrontar con autonomía y responsabilidad todos los aspectos de la vida (personal, social, familiar y profesional) se romperán los esquemas de dominación y sumisión y mujeres y hombres podrán construir relaciones interpersonales y relaciones afectivas en igualdad.

Y la escuela puede tener un papel fundamental en esta tarea. ¡Animémonos a abordarla! 


\section{Bibliografía}

AGIRRE SÁEZ DE EGUILAZ, Ana, "Chicos y chicas imaginan el futuro". Cuadernos de Pedagogía 257 (1997), pp. 85-89.

- "La coeducación como marco para la prevención de la violencia". Aula de Innovación Educativa 177 (2008), pp. 23-25.

BONAL, Xabier y TOMÉ, Amparo, Construir la escuela coeducativa: la sensibilización del profesorado. Barcelona: Universidad Autónoma de Barcelona, Instituto de Ciencias de la Educación, 1997.

EMAKUNDE, Guía de buenas prácticas PAREKO. Vitoria-Gasteiz: Emakunde/ Instituto Vasco de la Mujer, 2000.

FISAS, Vicent, El sexo de la violencia: Género y cultura de la violencia. Barcelona: Icaria, 1998.

INSTITUTO DE LA MUJER: Prevenir la violencia. Una cuestión de cambio de actitud. Madrid: Instituto de la Mujer, 1998.

JIMÉNEZ ARAGONÉS, Pilar, Materiales didácticos para la prevención de la violencia de género: Unidad didáctica para Educación Primaria. Sevilla: Junta de Andalucía, Consejería de Educación y Ciencia, Instituto de la Mujer, 1999.

MARTÍN SERRANO, Esperanza y MARTÍN SERRANO, Manuel, Las violencias cotidianas cuando las víctimas son mujeres. Madrid: Instituto de la Mujer, 1999.

MIEDZIAN, Myriam, Chicos son, hombres serán: ¿Cómo romper los lazos entre masculinidad y violencia. Madrid: Horas y horas, 1996.

SANTOS, Miguel Ángel (coord.), El harén pedagógico. Barcelona: Grao, 2000.

BLANCO, Nieves, Educar en femenino y en masculino. Madrid: Akal, 2001.

SUBIRATS, Marina y TOMÉ, Amparo, Balones fuera: reconstruir los espacios desde la coeducación. Barcelona: Octaedro, 2007.

TOMÉ, Amparo, "Los valores que sustentan la coeducación". Perspectiva CEP 5 (2005).

TOMÉ, Amparo y RAMBLA, Xavier, Contra el sexismo: coeducación y democracia en la escuela. Madrid: Síntesis, 2001.

URRUZOLA, Ma José, ¿Es posible coeducar en la actual escuela mixta. Bilbao: Maite Canal, 1991.

V.AA., Mujer y educación: educar para la igualdad, educar desde la diferencia. Barcelona: Grao, 2002. 